\title{
THE SCALABLE COHERENT INTERFACE, IEEE P1596, STATUS AND POSSIBLE APPLICATIONS TO DATA ACQUISITION AND PHYSICS*
}

\author{
David B. Gustayson \\ Stanford Linear Accelerator Center, Stanford Uninersity, Stanford. CA 94909
}

\section{Abstract}

IEEE P1596, the Scalable Coherent Interface (formerly known as SuperBus) is based on experience gained while developing Fastbus (ANSI/IEEE 960-1986, IEC 935). Fulurebus (IEEE P896.x) and other modem 32-bit buses. SCI goals include a minimum bandwidth of $1 \mathrm{GByte} / \mathrm{sec}$ per processor in multiprocessor systems with thousands of processors; efficient support of a coherent distributed-cache image of distributed shared memory; support for repeaters which interface to existing or future buses; and support for inexpensive small rings as well as for general switched interconnections like Banyan, Omega, or crossbar networks.

This paper presents a summary of current directions. reports the status of the work in progress, and suggests some applications in data acquisition and physics.

\section{INTRODUCTION}

\section{A. A Computing-Industry Problem}

The coming generation of supercomputers-on-a-chip has raised computation price/performance expectations, wreaking havoc in the supercomputer industry. However, actually delivering the performance these chips promise requires new supporting infrastructure.

Several supercomputer manufacturers have gone out of business recently; the reason is certainly not lack of demand for computation, but rather that supercomputer mainframes do not appear to be cost-effective when compared with warkstations or network compute servers. (However, some problems really da require the resources of a supercomputer.)

In order to produce a successful supercomputer, one has to develop new technology. Unfortunately, this is dangerously unpredictable, expensive and time consuming. By the time the technology is debugged and the final machine is ready to deliver, the faster-moving integrated circuit technology has produced processors which are so fast that a thousand dullars worth of new chjps promises a significant fraction of the throughput of the multi-million-dollar supercomputer. The supercomputer can only survive because of the valuable software infrastructure which supports it, and because of a small number of customers who absolutely need these biggest machines regardless of the price.

\footnotetext{
* Work supported by the Department of Energy, contract DE-ACO376 SFOOS15.
}

\section{B. Solution Strategy}

A way is needed to harness multiple inexpensive fast processor chips and apply them to large problems. A large part of this problem is software. Enormous effort has been applied to this problem over the last decade or so. and progress has been made; the problem is not trivial, but it is not insurmountable either. I will noi discuss the software here, but consider only the hardware aspects of the problem (while keeping in mind that hardware must provide certain facilities to make the software protlems manageable).

Suppose that the interface between the processor part and the rest of a (large) system could be standardized. Then a supercompuier manufacturer would spend its effort on 'developing an efficient interconnect (switch), $1 / 0$, cooling. power system, and mulaprocessor softwane. All these are technologies which change less rapidly than processor chip technology, and are not susceptible to quick obsolescence. When everything etse is ready, the manufacturer would buy a large number of the latest standard processor boards and plug them in. Though this is a slight oversimplification (e.g., part of the system software probably does care about detuils of the processor chip). I think it represents a strategy which is becoming practical now, and greally improves the chance of producing a cost-effective marketable product.

\section{Solution Implementation}

IEEE P1596, Scalable Coherent Interface, aims to be exactly the needed standard interface betwren processor and interconneet. SCI standardizes the physical characteristics of the processor board and connector, the electrical signalling. power distribution, and signalling protocols. In addition, SCI will define a fiber optic implementation of the protocols (SCIFI) which operates at lower speeds (due to current fiber-optic technology limitations) but over longer distances, and a personal-computer form factor for those who might like deskıop gigaflops.

In fact, though I introduced $\mathrm{SCl}$ in the context of supercomputers here, an important aspect of SCl's design is its suitability for use in inexpensive small compulers. It is the high volume production which can be supported by the smallcomputer market which we all need in order w bring the prices down for the large-computer market. One of the most attractive aspeces of SCI is the smooth growth palh it provides. allowing buyers to expand their systems as future needs require, while preserving their initial investrment. 


\section{THE SCI SOLUTION}

\section{A. Hardware Architecture}

Computer bus structures cannot cope with the high speeds that will soon be reeded to suppor fast integraled processors. In addition to the electrical problems, buses inherently become bottlenecks because they are shared resources. Instead, SCI uses a large number of independent unidirectional links. More explanation of the problems of buses is available in the references.

Since cac: SCI node has one input and one output link, the protocols have to provide for the output data flow to be controlled somehow by the input data. This implies a loop or ring of some sor in order to provide the necessary feedback. This ring might be a large simple one, where several modules are connected output link to input link, or a trivial one with two modules, one of which is really the interface to a hidirectional switch. In turn, the switch might be made of a mesh of rings connected by bridging nodes. It is casy to generate butterfly-like switch characteristics in this way, and also more complex switches.

Thus the inherent association of SCI with ring struclures does not appear to be a serious limitation. Other switch-based machines have to provide feedback in some way, 100 . Except for some which operate synchronously (and thus have inherent speed and size limits), the main difference is that $\mathrm{SCl}^{\prime} \mathrm{s}$ feedback path is full bandwidth, where others have instead added a special low-bandwidth reverse signal on each lin' That introduces scaling problems and makes signal regeneration more difficulc.

Even a single SCI ring, with up 10 (say) eight processors, will far outperform a bus because of the high bandwidth and fast arbitration and addressing mechanisms (cache controllers on buses often slow address cycles for all when the direciories are busy because of on-board processor activity). SCI's cost should also be much lower, because of the narrow interface and the elimination of complex sncoping cache controllers.

Unidirectional links effectively remove the speed-of-light barrier to system growth: the system size and clock rate are decoupled, and there are no cycle-by-cycle handshakes. Physical signalling problems are greatly simplified because there is always one driver and one receiver, at opposite ends of the link. Signads operate steadily whether there is data flowing or not, which makes it easy to use phase locked loops for data extraction if desired (no start-up preamble is required).

\section{B. Signalling}

Differential ECL (Emitter Coupled Logic) signalling works well at SCI rates. It rejects noise, survives atlenuation, and results in constant current flow between connected modules, enormously simplifying the ground distribution problem compared to bussed systems.
SCI uses a narnow 16-bit data path (plus clock and one flag bii) at 2 as/word ( $250 \mathrm{MHz}$ clock, both edges active), to control the interface IC pin-count problim and make switch elements more practical. Note that 'differential' implies 2 pins per signal, and 'unidirectional' implies 2 links, one for inpul and one for output, so we have 72 pins for cach SCI interface. A circuit for making switch networks must have at least twice that many, and preferably four or eight times, so the impontance of a narrow dala path becomes obvious.

In this signalling scheme, the practical limits on signal transmission become attenuation, distortion, and skew. The nature of the unidirectional link makes it easy to regeneratc and re-lime signals with simple repeater circuits as needed along a cable. Preliminary experiments have transmitted data 6 meters on inexpensive ribbon cable without repeaters. This did not appear to be near the practical limit: however, ewisted pair and shielded flat cables did not work as well - highfrequency behavior of the cable is important.

The SCI protocols include occasional patterns which reveal bit boundaries for dynamic skew compensation. The receiving circuit provides an on-chip delay chain, and swilches delay in or out on each bit until the observed skew is effectively eliminated. It is not yet certain whether the initial chips will inclu de this circuitry, as it does not appear to be essential and there is compelition for available gates. When such chips become available, however, inexpensive cabling can be uscd and the skew changes which occur as it is moved or handled can be dynamically compensaved.

\section{Clocking}

The SCI interface is synchronous, but the phase of the incoming data stream with respect to the local clock depends on propagation delays and clock frequency differences and thus is arbilsary. Receiver circuits insert delay before sampling the data in order to synchronize it to the local clock.

We support several models of clock distribution. In the simplest, one can use a central clock as a $250 \mathrm{MHz}$ reference so that phase differences are constant. This is inconvenient to manage, however, in large systems or when reconfiguring small ones (e.g., when adding a second subrack or crate), and is completely impractical in Fiber-SCI syslems. Therefcre. we specify a mechanism which allows each interface to generale its own clock, and we insen or delete elasticity symbols as needed to compensate for differing clock rates.

Much of this compensating mechanism would have been needed in any case, as it is critical 10 avoid sampling the incoming data too near its transitions in order to avoid trjggering metastable states in the sampling circuits. Thus we have to observe the incoming clock phase relative to our ow/n clock and adjust the sampling time to account for drifts. Small systems can use the input link's clock at cach node, regenerating it fo. output to the next node, but care must be taken to avoid excessive buildup of phase jitter. 


\section{Connector}

Modelling and oxperiments have shown that several commercial connectors are able to meet our requirements. We intend to follow Futurcbust in adopting the DuPont Metral $2 \mathrm{~mm}$ conncctor, in a $4 \times \mathbf{4 8}$ size. The pinout is organized as a row of differential cutputs, a row of grounds, a row of differential inputs, and a row of differential stauc signals such as geographic address. Al one end of the connector we allocate 24 pins for $48 \mathrm{~V}$ supply, $48 \mathrm{~V}$ retum, power control, prechargc, and electrostatic discharge. There is enough extra room to isolate the power pins by leaving blank columns. Shor pins for the power control ensure that litte current is being drawn when a module is inserted or removed.

Tests reveaied that the card-edge connector used in IBM's PS/2 MicroChannel has excellent electrical characteristics. A variant of this, which automatically connects the input link to the output link when the board is removed, is being considered for the PC version. Thus empty sockets do not break the ring.

We were dismayed to learn that existing bus standards can be unreliable because they only define the gross mechanical shape of the connecior, not the pin construction or metallurgy. Even specifying the normal forces or insertion forces is not sufficient. In particular, the common situation where half of the mating pair is made by Ge vendor and half by another can lead to wially unsatisfactory performance. We plan to follow the lead of IBM reliability researchers, and specify the shape of one side, the mecallurgy, and the stress which should be applied by the other side. This should result in long-tom reliable operation even if muluple vendors are involved.

\section{E. Power}

SCI specifics on-board DC/DC power conversion. This makes power-on insertion and removal of modules easy. which can be very important in large systems. (When multiple voltages are supplied to a module through its connector, it is very dificult to guarantec a safe sequence of power-contact making or breaking as modules are inserted or removed.) Also, uninterruptable power is almost trivially simple to provide in the contexl of on-board conversion.

Furtherrtorc, it is not clear which stpply voluges will be important in luture designs; ECL wants $-2 \mathrm{~V}$ and $-5 \mathrm{~V}$. TTL and CMOS want $+5 \mathrm{~V}$, and $3.3 \mathrm{~V}$ or lower seems important for future CMOS and GaAs technologies. On-board conversion avoids guessing future requirements, and enormonsly reduces the number of power pins which would necd to be allocaled to provide for full current at each of several potentially useful supply voltages.

The primary disadvantages of on-board conversion are the convener's spuce and auded heat load on the board. Though modern converter technology has reduced this problem considerably, it still causes us to specily a somewhat larger board than would otherwisc be required.

\section{F. Board and Module}

We have chosen to specify a single board size, the Eurocard GU $(233.35 \mathrm{~mm}) \times 280 \mathrm{~mm}$. The module width, or pitch, is 25.4mm. Boards can use side-mounted or straddlemounted connectors, providing for double-sided surface mount or single-sided tall components, at the cost of dual card guides or moveable card guides.

The mechanies for a whole related family of board sizes and corresponding backplanes and subracks is tetailed in IEEE P1 101.2, an adaptation of IEEE 1101 but using the new $2 \mathrm{~mm}$ cornector family. Development of a new all-metric standard is under way (IEEE P1301.1), and we suppon that in printiple, but won't wait for it to be completed.

\section{G. Protocols}

$\mathrm{SCl}$ has been careful to keep its protocols lean and efficient. All transactions are 'split', with a request subaction followed by a response subaction. A small set of transactions has been defined which permit fixed length transfers of 0,64 and 256 byles, variable lengths from 1-16 byles, and several special transactions associated with coherence list maintenance and generalized multiprocessor tocks. Fjxed length packets simplify the very fast logic required in the $\mathrm{SCI}$ interface chips. Block transfers are short enough to limit switch blockage, while providing reasonable data transfer efficiency. All packets are a multiple of 8 bytes in order to facilitate intemal demuluplexing so that stower but wider anchip logic paths can be used.

We have included several forward-progress mechanisms in order to prevent starvation of certain nodes:

1. Our arbitration protocols guarantee fair access to a small fraction of the system bandwidth (important for guarantees of forward progress), with optional priority allocation of the rest.

2. A batched retry mecharism guarantees fair access to a saturated node.

To avoid certain common kinds of deadlock, we specify separate requesi and response queues, and forbid mechanisms in which responses generate dependent requests. This breaks a cyclic dependency which otherwise could severely impact system performance. (E.g., with combined queues all entries could be filled with requests, making resporsus impossible.)

\section{H. Coherence}

We have developed a basic cache coherence mechanism which maintains a distributed directory of users of each data item (64-byte cache line), so that oniy those who care have to be notified when shared data is modilied. By storing this directory as linked-list pointers in cach participating cache, the storage requited does not have to be preallocated and there is no intrinsic limit to growth. This mechanism appears fairly 
simple at first, but the hazards can be subule, as it involves maintaining a correct distributed structure which is built and modified by many processors, potentially at the same time. We hope to verify the correctness of this mechanism by formal means[2] as well as by careful design and simulation tests.

Externsions to this basic mechanism are being developed which may approach the ultimate $\mathrm{N} / \mathrm{log} \mathrm{N}$ performance desired for very large systems. Request combining allows reduction of traffic at system hotspots, and approximate tree pointers allow spreading information to sublists faster than linear lists permit, while avoiding the overheads associated with maintaining balanced urees.

Efficient muliprocessor locks have been developed. Most of these are needed mainly for controlling the interface to existing buses. For multiprocessor operation in a coherent SCI domain, specific lock operations are not needed: the processor exclusively locks a cache line briefly and does what it wishes.

SCI emphasizes coherent cache/shared memory operation only because that is the difficult service to provide, and is the most general processing model. However, coherent and noncoherent operation can coexist, along with message passing. for programs which work well in those simpler environments.

\section{PHYSICS APPLICATIONS}

Technical work often includes large compulations. For example, airframe designers would like teraflop machines for studying aerodynamic behavior without using wind tunnels. Tomographic imaging, which involves deducing internal structure based on the observation of many projections, is compule intensive. Real-time 3-D Magnetic Resonance Imaging involves enomous data handling problems.

Modern theoretical physicists are often compute-limited. Currently, dedicated multiprocessors are being used for latticeguage-Lheory calculations at Fermilab, for example.

Multiprocessor farms have been buitt at many particle physics laboratories to provide cost-effective analysis for the enormous volumes of experimental data. The software problem for particle physics event analysis is paricularly easy, becanse the events are statistically independent of one another. one merely distributes them to independent processors for analysis, and combines the results later.

Data production at the Superconducting SuperCollider is estimated at oyer $10^{14}$ bytes/second, with enormous computation problems associated with triggcring, filtering, storing and analyzing the data. At these data rates, the aforementioned independence of events breaks down because of detector time constants, so analysis may require examination of one or more events preceding the given event. This will probably require enormously increased interprocessor communication. SCI's coherence mechanisms may be helpful in managing this problem, where simple message-passing was adequate in the past The ability of the $\mathrm{SCl}$ architecture to grow seamlessly as budgets permit will be a great convenience as well.

SCl's fiber-optic implementation mas hi saeful for moving data oul of detectors. This uses little of SCI's special capabilities, but there may be some advalitage to having the data arrive in SCI-format packets if SCI is to be used in the data filtering and analysis system.

\section{CONCLUSION}

The SCI project has met its milestones on schedule, strongly motivated by its commercial implementors. The current plan aims for an approved standard covering the physical signalling, logical protocols, and cache coherence mechanism in 1990 . The first commercial implementor (Dolphin Server Technology, Oslo, Norway) expects to have working prototypes within a few months of the standard's approval, and has promised to make the interface chips available to others.

SCI has no evident competition in terms of open systems which could hope 10 deal with the massive computation needs for the next generation of data acquisition, analysis, and general computation.

For details, or to participate, please contact the author:

David B. Gustavson, EEEE P1596 Chaiman

Computation Reseanch Group, bin 88

Stanford Linear Accelerator Center

Stanford, CA 94309 U.S. A.

tel: (415) 926-2863 fax: (415) 323-3626

bitmet: DBG@SLACVM

\section{REFERENCES}

[1] K Ahes, E. H. Kristiansen, D. B. Gustavson, D. V. James, "Scalahle Coherent Interface", CompEuro 90, Tel Aviv, Irael, May 1990.

[2] S. Gjessing. S. Krogdahl, E. Munthe-Kaas, "Formal Specification and Vefification of SCl Cache Coherence". NIK89. Stavenger, Norway, November 1989.

B] D. B. Gustavson, "Scalable Coherent Interface". COMPCON Spring 1989. Sen Francisco, CA, February 27-March 3, 1989.

[4] D. B. Gustavsor, "IEEE P1596, A Scalable Coherent Interface for Gigabyle/sec Multiprocessor Applications", Nuclear Science Symposium, Otlando, Florida, November 9-11, 1988.

[5] D. V. James, "Scalable IO Architecture for Buses", COMPCON Spring 1989, Sm Francisco, CA, Februery 27-March 3, 1989.

[6] E. H. Kristiansen, K. Alnes, B. O. Bakka and M. Jenssen, "Scalable Coherent Isierface", Eurobus Munich, May 1989.

[7] P. Swenzey, "Cache Coherence on SCI", IEEE/ACM Computer Anchitecture Workshop. Eilath Istacl, June 1989. 


\section{DISCLAIMER}

This report was prepared as an account of work sponsored by an agency of the United States Government. Neither the United States Government nor any agency thereof, nor any of their employees, makes any warranty, express or implied, or assumes any legal liability or responsibility for the accuracy, completeness, or usefulness wi any information, apparatus, product, or process disclosed, or represents that its use would not infringe privately owned rights. Reference herein to any specilje commercial product, process, or service by trade name, trademark, manufacturer, or otherwise does not necessarily constitute or imply its endorsement, recommendation. or favoring by the Unied States Government or any agency thereof. The views and opinions of authors expressed herein do not necessarily state or reflect those of the United States Government or any agency thereof. 